\title{
On Certain Subclasses of Analytic and Univalent Functions based on an Extension of Salagean Operator
}

\author{
T.V. Sudharsan and R. Vijaya
}

\begin{abstract}
There is many subclasses of analytic and univalent functions. A class $T$ of functions with negative coefficients introduced by Silverman [8] opened up a new and fruitful line of research in the theory of univalent functions. Following the works of Khairnar and Meena More [3], Aghalary and Kulkarni [1], Silverman and Silvia [8] and Owa and Nishiwaki [5] on analytic and univalent functions, in this paper we introduce two new classes for a family of analytic function with negative coefficients. We have attempted to obtain coefficient estimate, distortion theorem and extreme points for the class $\operatorname{TSn}(\alpha, \beta, \xi, \gamma)$ and $\operatorname{TKn}(\alpha, \beta, \xi, \gamma)$ for $|z|<$ $1,0<\beta \leq 1,{ }^{\frac{1}{2}} \leq \xi \leq 1,0 \leq \alpha \leq \leq^{\frac{1}{2}} \xi$ and $^{\frac{1}{2}}<\gamma \leq 1$.
\end{abstract}

Keywords--- Convex Functions, Salagean Operator, Starlike Functions, Univalent Functions

\section{INTRODUCTION}

$\mathrm{T}$ HE classical field of analytic univalent function of a complex variable which is classified under geometric function theory. Due to interesting connections with geometry and analysis continues to attract active research by function theorists.

In the investigation of an analytic function there resulted a new direction of research in this theory namely, construction of subclasses of analytic univalent function is trying to settle a well known conjecture known as the Bieberbach conjecture which was positively settled by Branges [2] in 1985.

Let A denote the class of functions given by

$$
f(z)=z+\sum_{j=2}^{\infty} a_{j} z^{j}
$$

Which are analytic in the unit disc E: $\{\mathrm{z}:|\mathrm{z}|<1\}$ and normalized by $\mathrm{f}(0)=0, \mathrm{f}^{\prime}(0)=1$ and let $\mathrm{S}$ be the subclass of A consisting of analytic and univalent functions of the form (1.1).

We denote by $S^{*}(\alpha)$, K $(\alpha)$, consisting of all functions which are, respectively star like and convex of order $\alpha$ in $\mathrm{E}$ with $0 \leq \alpha<1$. Thus

T.V. Sudharsan, Department of Mathematics, SIVET College, Chennai-600073, India.E-mail: tvsudharsan@ rediffmail.com

R. Vijaya, Department of Mathematics, S.D.N.B. Vaishnav College, Chennai - 600 044, India.E-mail: viji_dorai67@yahoo.co.in

$$
\begin{gathered}
S^{*}(\alpha)=\left\{f \in S: \operatorname{Re}\left(\frac{z f^{\prime}(z)}{f(z)}\right)>\alpha: 0 \leq \alpha<1, z \in E\right\} \\
K(\alpha)=\left\{f \in S: \operatorname{Re}\left(1+\frac{z^{\prime \prime}(z)}{f^{\prime}(z)}\right)>\alpha: 0 \leq \alpha<1, z \in E\right\} .
\end{gathered}
$$

The subfamily $\mathrm{T}$ of $\mathrm{S}$ consists of functions of the form

$$
f(z)=z-\sum_{j=2}^{\infty} a_{j} z^{j} \quad\left(a_{j} \geq 0\right)
$$

For functions $f(z) \in S$, Salagean [6] introduced a new differential operator called Salagean differential operator $D^{n}$ defined by $\mathrm{D}^{\mathrm{n}}: \mathrm{A} \rightarrow \mathrm{A}, \mathrm{n} \in \mathrm{N}$

$$
\begin{aligned}
& D^{0} f(z)=f(z) \\
& D^{1} f(z)=D f(z)=z^{\prime}(z) \\
& \quad \vdots \\
& D^{n} f(z)=D\left[D^{n-1} f(z)\right], \quad n \in N
\end{aligned}
$$

The basic classes of convex functions, starlike functions, functions with negative coefficients and many other introduced and several properties like the coefficient problem, radius of convexity problem and so on have been investigated.

In this paper using Salagean operator $\mathrm{D}$, we introduce the classes $\mathrm{TS}_{\mathrm{n}}(\alpha, \beta, \xi, \gamma)$ and $\mathrm{TK}_{\mathrm{n}}(\alpha, \beta, \xi, \gamma)$ and obtain sharp results for coefficient estimates. This leads to extremal properties. We also obtain growth and distortion theorem.

Definition 1.1 A function $\mathrm{f} \in \mathrm{T}$ is said to be in the class $\operatorname{TS}_{\mathrm{n}}(\alpha, \beta, \xi, \gamma)$ if and only if

$$
\left|\frac{\frac{D^{n+1} f(z)}{D^{n} f(z)}-1}{2 \xi\left(\frac{D^{n+1} f(z)}{D^{n} f(z)}-\alpha\right)-\gamma\left(\frac{D^{n+1} f(z)}{D^{n} f(z)}-1\right)}\right|<\beta
$$

For $|z|<1,0<\beta \leq 1, \frac{1}{2} \leq \xi \leq 1,0 \leq \alpha \leq \frac{1}{2} \xi$ and $\frac{1}{2}<\gamma \leq$ 1.

If $\mathrm{n}=0$, we obtain the corresponding result of Khairnar and Meena More [3].

If we replace $\gamma$ by $1, \mathrm{n}=0$, in the above inequality, we obtain the results of Aghalary and Kulkarni [1] and Silverman and Silvia [8].

If we replace $\xi$ by $1, \mathrm{n}=0$, we obtain the results of Owa and Nishiwaki [5]. 
Furthermore, a function $\mathrm{f}(\mathrm{z})$ is said to belong to the class $\operatorname{TK}_{\mathrm{n}}(\alpha, \beta, \xi, \gamma)$ if and only if $\mathrm{zf}^{\prime}(\mathrm{z}) \in \mathrm{TS}_{\mathrm{n}}(\alpha, \beta, \xi, \gamma)$.

II. COEFFICIENT ESTIMATES AND OTHER PROPERTIES

Theorem 2.1 Let $\mathrm{f}$ be defined by

$$
f(z)=z-\sum_{j=2}^{\infty} a_{j} z^{j} \quad\left(a_{j} \geq 0\right)
$$

is in $\mathrm{TS}_{\mathrm{n}}(\alpha, \beta, \xi, \gamma)$ if and only if

$$
\sum_{j=2}^{\infty}\left[\left(j^{n+1}-j^{n}\right)-\beta\left(2 \alpha \xi j^{n}-2 \xi j^{n+1}+\gamma j^{n+1}-\gamma j^{n}\right)\right] a_{j} \leq 2 \beta \xi(1-\alpha)
$$

Proof Suppose

$$
\sum_{j=2}^{\infty}\left[\left(j^{n+1}-j^{n}\right)-\beta\left(2 \alpha \xi j^{n}-2 \xi j^{n+1}+\gamma j^{n+1}-\gamma j^{n}\right)\right] a_{j} \leq 2 \beta \xi(1-\alpha)
$$

We have

$$
\begin{aligned}
& \left|D^{n+1} f(z)-D^{n} f(z)\right| \\
& \quad-\beta\left|2 \xi\left(D^{n+1} f(z)-\alpha D^{n} f(z)\right)-\gamma\left(D^{n+1} f(z)-D^{n} f(z)\right)\right|<0
\end{aligned}
$$

With the provision

$$
\begin{aligned}
& \mid \sum_{j=2}^{\infty}\left[\left(j^{n+1}-j^{n}\right) a_{j} z^{j} \mid\right. \\
& \quad-\beta\left|2 \xi(1-\alpha) \sum_{j=2}^{\infty}\left(2 \alpha 2 j^{n}-2 \xi j^{n+1}+\gamma j^{n+1}-\gamma j^{n}\right) a_{j} z^{j}\right|<0
\end{aligned}
$$
by

For $|z|=r<1$, then the condition (2.2) is bounded above

$$
\begin{aligned}
& \sum_{\mathrm{j}=2}^{\infty}\left(\mathrm{j}^{\mathrm{n}+1}-\mathrm{j}^{\mathrm{n}}\right) \mathrm{a}_{\mathrm{j}} \mathrm{r}^{\mathrm{j}}-2 \beta \xi(1-\alpha) \\
& \quad-\beta \sum_{\mathrm{j}=2}^{\infty}\left(2 \alpha 2 \mathrm{j}^{\mathrm{n}}-2 \xi \mathrm{j}^{\mathrm{n}+1}+\gamma \mathrm{j}^{\mathrm{n}+1}-\gamma \mathrm{j}^{\mathrm{n}}\right) \mathrm{a}_{\mathrm{j}} \mathrm{r}^{\mathrm{j}} \\
& =\sum_{\mathrm{j}=2}^{\infty}\left[\left(\mathrm{j}^{\mathrm{n}+1}-\mathrm{j}^{\mathrm{n}}\right)-\beta\left(2 \alpha \xi \mathrm{j}^{\mathrm{n}}-2 \xi \mathrm{j}^{\mathrm{n}+1}+\gamma \mathrm{j}^{\mathrm{n}+1}-\gamma \mathrm{j}^{\mathrm{n}}\right)\right] \mathrm{a}_{\mathrm{j}} \mathrm{r}^{\mathrm{j}} \\
& \quad-2 \beta \xi(1-\alpha) \\
& \leq \sum_{\mathrm{j}=2}^{\infty}\left[\left(\mathrm{j}^{\mathrm{n}+1}-\mathrm{j}^{\mathrm{n}}\right)-\beta\left(2 \alpha \xi \mathrm{j}^{\mathrm{n}}-2 \xi \mathrm{j}^{\mathrm{n}+1}+\gamma \mathrm{j}^{\mathrm{n}+1}-\gamma \mathrm{j}^{\mathrm{n}}\right)\right] \mathrm{a}_{\mathrm{j}}-2 \beta \xi(1-\alpha) \\
& \leq 0 \quad \sum_{\mathrm{j}=2}^{\infty}\left[\left(\mathrm{j}^{\mathrm{n}+1}-\mathrm{j}^{\mathrm{n}}\right)-\beta\left(2 \alpha \xi \mathrm{j}^{\mathrm{n}}-2 \xi \mathrm{j}^{\mathrm{n}+1}+\gamma \mathrm{j}^{\mathrm{n}+1}-\gamma \mathrm{j}^{\mathrm{n}}\right)\right] \mathrm{a}_{\mathrm{j}} \leq 2 \beta \xi(1-\alpha) \\
& \therefore \mathrm{f}(\mathrm{z}) \in \mathrm{TS}_{\mathrm{n}}(\alpha, \beta, \xi, \gamma) .
\end{aligned}
$$

Conversely, let $\mathrm{f}(\mathrm{z}) \in \mathrm{TS}_{\mathrm{n}}(\alpha, \beta, \xi, \gamma)$.

Then from the definition of $f(z)$, we have

$$
\left|\frac{\frac{D^{n+1} f(z)}{D^{n} f(z)}-1}{2 \xi\left(\frac{D^{n+1} f(z)}{D^{n} f(z)}-\alpha\right)-\gamma\left(\frac{D^{n+1} f(z)}{D^{n} f(z)}-1\right)}\right|<\beta
$$

that is,

$\left|\frac{\sum_{j=2}^{\infty}\left(j^{n+1}-j^{n}\right) a_{j} z^{j}}{2 \xi\left[z(1-\alpha)+\sum_{j=2}^{\infty}\left(\alpha j^{n+1}-j^{n}\right) a_{j} z^{j}\right]-\gamma \sum_{j=2}^{\infty}\left(j^{n+1}-j^{n}\right) a_{j} z^{j}}\right|<\beta$

As $|\operatorname{Re} \mathrm{f}(\mathrm{z})| \leq|\mathrm{z}|$ for all $\mathrm{z}$, we have

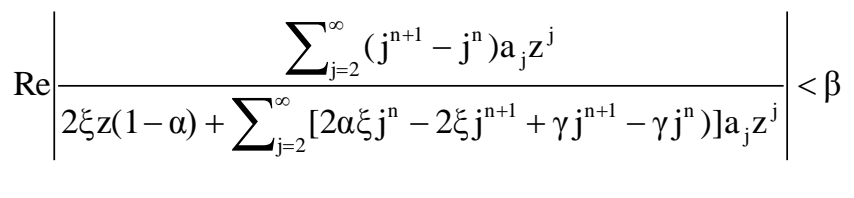

we choose the values of $z$ on real axis, such that $\frac{D^{n+1} f(z)}{D^{n} f(z)}$ is real and upon clearing, the denominator of the above expression and allowing $\mathrm{z} \rightarrow 1$ through real values, we obtain

$$
\begin{aligned}
\sum_{j=2}^{\infty}\left[\left(j^{n+1}-j^{n}\right)-\beta\left(2 \alpha \xi j^{n}-2 \xi j^{n+1}+\gamma j^{n+1}-\gamma j^{n}\right)\right] a_{j} \\
-2 \beta \xi(1-\alpha) \leq 0 \\
\Rightarrow \sum_{j=2}^{\infty}\left[\left(j^{n+1}-j^{n}\right)-\beta\left(2 \alpha \xi j^{n}-2 \xi j^{n+1}+\gamma j^{n+1}-\gamma j^{n}\right)\right] a_{j}
\end{aligned}
$$$$
\leq 2 \beta \xi(1-\alpha) \text {. }
$$

Remark 2.1. If $\mathrm{f}(\mathrm{z}) \in \mathrm{TS}_{\mathrm{n}}(\alpha, \beta, \xi, \gamma)$, then

$\mathrm{a}_{\mathrm{j}} \leq \frac{2 \beta \xi(1-\alpha)}{\sum_{\mathrm{j}=2}^{\infty}\left[\left(\mathrm{j}^{\mathrm{n}+1}-\mathrm{j}^{\mathrm{n}}\right)-\beta\left(2 \alpha \xi \mathrm{j}^{\mathrm{n}}-2 \xi \mathrm{j}^{\mathrm{n}+1}+\gamma \mathrm{j}^{\mathrm{n}+1}-\gamma \mathrm{j}^{\mathrm{n}}\right)\right]}$

$a_{j} \leq \frac{2 \beta \xi(1-\alpha)}{\left(j^{n+1}-j^{n}\right)-\beta\left(2 \alpha \xi j^{n}-2 \xi j^{n+1}+\gamma j^{n+1}-\gamma j^{n}\right)}$ for $j=2,3, \ldots$

and equality holds for

$$
f(z)=z-\frac{2 \beta \xi(1-\alpha)}{\left(j^{n+1}-j^{n}\right)-\beta\left(2 \alpha \xi j^{n}-2 \xi j^{n+1}+\gamma j^{n+1}-\gamma j^{n}\right)} z^{j} .
$$

Corollary 2.1. If $\mathrm{f}(\mathrm{z}) \in \mathrm{TS}_{\mathrm{n}}(\alpha, \beta, \xi, 1)$

$a_{j} \leq \frac{2 \beta \xi(1-\alpha)}{\left(j^{n+1}-j^{n}\right)-\beta\left(2 \alpha \xi j^{n}-2 \xi j^{n+1}+j^{n+1}-j^{n}\right)}$ for $j=2,3, \ldots$ and equality holds for

$$
f(z)=z-\frac{2 \beta \xi(1-\alpha)}{\left(j^{n+1}-j^{n}\right)-\beta\left(2 \alpha \xi j^{n}-2 \xi j^{n+1}+j^{n+1}-j^{n}\right)} z^{j}
$$

Corollary 2.2. If $\mathrm{f}(\mathrm{z}) \in \mathrm{TS}_{\mathrm{n}}(\alpha, \beta, 1,1)$,

$$
a_{j} \leq \frac{2 \beta(1-\alpha)}{\left(j^{n+1}-j^{n}\right)-\beta\left(2 \alpha j^{n}-j^{n+1}-j^{n}\right)} \text { for } j=2,3, \ldots
$$

And equality holds for 


$$
f(z)=z-\frac{2 \beta \xi(1-\alpha)}{\left(j^{n+1}-j^{n}\right)-\beta\left(2 \alpha j^{n}-j^{n+1}-j^{n}\right)} z^{j}
$$

Corollary 2.3 If $\mathrm{f}(\mathrm{z}) \in \mathrm{TS}_{\mathrm{n}}(\alpha, 1,1,1)$,

$$
\mathrm{a}_{\mathrm{j}} \leq \frac{(1-\alpha)}{\left(\mathrm{j}^{\mathrm{n}+1}-\alpha \mathrm{j}^{\mathrm{n}}\right)}
$$

Theorem 2.2 A function $f(z)=z-\sum_{j=2}^{\infty} a_{j} z^{j}, \quad\left(a_{j} \geq 0\right)$ is in $\operatorname{TK}_{\mathrm{n}}(\alpha, \beta, \xi, \gamma)$ if and only if

$$
\begin{aligned}
& \sum_{j=2}^{\infty} j^{n+1}\left[\left(j^{n+1}-j^{n}\right)-\beta\left(2 \alpha \xi j^{n}-2 \xi j^{n+1}+\gamma j^{n+1}-\gamma j^{n}\right)\right] a_{j} \\
& \leq 2 \beta \xi(1-\alpha)
\end{aligned}
$$

Proof The proof of this theorem is analogous of Theorem 2.1 , because a function $\mathrm{f}(\mathrm{z}) \in \mathrm{TK}_{\mathrm{n}}(\alpha, \beta, \xi, \gamma)$ if and only if $\mathrm{zf}^{\prime}(\mathrm{z}) \in \mathrm{TS}_{\mathrm{n}}(\alpha, \beta, \xi, \gamma)$, so it is enough that $\mathrm{a}_{\mathrm{j}}$ is replaced by $\mathrm{j}^{\mathrm{n}+1} \mathrm{a}_{\mathrm{j}}$ in Theorem 2.1 .

Remark 2.2 If $\mathrm{f}(\mathrm{z}) \in \mathrm{TK}_{\mathrm{n}}(\alpha, \beta, \xi, \gamma)$, then

$$
\begin{array}{r}
a_{j} \leq \frac{2 \beta \xi(1-\alpha)}{j^{n+1}\left[\left(j^{n+1}-j^{n}\right)-\beta\left(2 \alpha \xi j^{n}-2 \xi j^{n+1}+\gamma j^{n+1}-\gamma j^{n}\right)\right]} \\
\text { for } j=2,3, \ldots
\end{array}
$$

and equality holds for

$$
f(z)=z-\frac{2 \beta \xi(1-\alpha)}{j^{n+1}\left[\left(j^{n+1}-j^{n}\right)-\beta\left(2 \alpha \xi j^{n}-2 \xi j^{n+1}+\gamma j^{n+1}-\gamma j^{n}\right)\right]} z^{j}
$$

Corollary 2.4. If $\mathrm{f}(\mathrm{z}) \in \mathrm{TK}_{\mathrm{n}}(\alpha, \beta, \xi, 1)$

$$
\begin{array}{r}
a_{j} \leq \frac{2 \beta \xi(1-\alpha)}{j^{n+1}\left[\left(j^{n+1}-j^{n}\right)-\beta\left(2 \alpha \xi j^{n}-2 \xi j^{n+1}+j^{n+1}-j^{n}\right)\right]} \\
\text { for } j=2,3, \ldots
\end{array}
$$

and equality holds for

$f(z)=z-\frac{2 \beta \xi(1-\alpha)}{j^{n+1}\left[\left(j^{n+1}-j^{n}\right)-\beta\left(2 \alpha \xi j^{n}-2 \xi j^{n+1}+j^{n+1}-j^{n}\right)\right]} z^{j}$

Corollary 2.5. If $\mathrm{f}(\mathrm{z}) \in \mathrm{TK}_{\mathrm{n}}(\alpha, \beta, 1,1)$

$a_{j} \leq \frac{2 \beta(1-\alpha)}{j^{n+1}\left[\left(j^{n+1}-j^{n}\right)-\beta\left(2 \alpha j^{n}-j^{n+1}-j^{n}\right)\right]}$ for $j=2,3, \ldots$ and equality holds for

$$
f(z)=z-\frac{2 \beta(1-\alpha)}{j^{n+1}\left[\left(j^{n+1}-j^{n}\right)-\beta\left(2 \alpha j^{n}-j^{n+1}-j^{n}\right)\right]} z^{j}
$$

Corollary 2.6. If $\mathrm{f}(\mathrm{z}) \in \mathrm{TK}_{\mathrm{n}}(\alpha, 1,1,1)$

$$
a_{j} \leq \frac{(1-\alpha)}{j^{n+1}\left[\left(j^{n+1}-\alpha j^{n}\right)\right]}
$$

\section{GROWTH AND DisTORTION THEOREM}

Theorem 3.1. If $\mathrm{f}(\mathrm{z}) \in \mathrm{TS}_{\mathrm{n}}(\alpha, \beta, \xi, \gamma)$, then

$$
\begin{aligned}
& \mathrm{r}-\frac{2 \beta \xi(1-\alpha)}{\left(2^{\mathrm{n}+1}-2^{\mathrm{n}}\right)-\beta\left(2 \alpha \xi 2^{\mathrm{n}}-2 \xi 2^{\mathrm{n}+1}+\gamma 2^{\mathrm{n}+1}-\gamma 2^{\mathrm{n}}\right)} \mathrm{r}^{2} \leq|\mathrm{f}(\mathrm{z})| \\
& \leq \mathrm{r}+\frac{2 \beta \xi(1-\alpha)}{\left(2^{\mathrm{n}+1}-2^{\mathrm{n}}\right)-\beta\left(2 \alpha \xi 2^{\mathrm{n}}-2 \xi 2^{\mathrm{n}+1}+\gamma 2^{\mathrm{n}+1}-\gamma 2^{\mathrm{n}}\right)} \mathrm{r}^{2} .
\end{aligned}
$$

Equality holds for

$$
f(z)=z-\frac{2 \beta \xi(1-\alpha)}{[(1+4 \beta \xi)-\beta(\gamma+2 \alpha \xi)]}
$$

Proof. By Theorem 2.1, we have $\mathrm{f}(\mathrm{z}) \in \mathrm{TS}_{\mathrm{n}}(\alpha, \beta, \xi, \gamma)$ if and only if

$$
\sum_{j=2}^{\infty}\left[\left(j^{n+1}-j^{n}\right)-\beta\left(2 \alpha \xi j^{n}-2 \xi j^{n+1}+\gamma j^{n+1}-\gamma j^{n}\right)\right] a_{j} \leq 2 \beta \xi(1-\alpha)
$$

or equivalently,

$$
\begin{gathered}
\sum_{\mathrm{j}=2}^{\infty}\left[\mathrm{j}^{\mathrm{n}+1}-\left(\mathrm{j}^{\mathrm{n}}-\frac{2 \beta \xi(1-\alpha) \mathrm{j}^{\mathrm{n}}}{1+2 \beta \xi-\gamma \beta}\right)\right] \mathrm{a}_{\mathrm{j}} \leq \frac{2 \beta \xi(1-\alpha)}{1+2 \beta \xi-\gamma \beta} \\
\text { Let } \mathrm{t}=\mathrm{j}^{\mathrm{n}}-\frac{2 \beta \xi(1-\alpha)}{1+2 \beta \xi-\gamma \beta} . \\
\mathrm{f}(\mathrm{z}) \in \mathrm{TS}_{\mathrm{n}}(\alpha, \beta, \xi, \gamma) \text { if and only if } \\
\sum_{\mathrm{j}=2}^{\infty}\left(\mathrm{j}^{\mathrm{n}+1}-\mathrm{t}\right) \mathrm{a}_{\mathrm{j}} \leq \mathrm{j}^{\mathrm{n}}-\mathrm{t} \quad(3.3)
\end{gathered}
$$

where $\mathrm{t}=\mathrm{j}^{\mathrm{n}}-\frac{2 \beta \xi(1-\alpha)}{1+2 \beta \xi-\gamma \beta}$

when $\mathrm{j}=2$,

$$
\begin{aligned}
\sum_{j=2}^{\infty}\left(2^{n+1}-t\right) a_{j} & \leq \sum_{j=2}^{\infty}\left(j^{n+1}-t\right) a_{j} \\
& \leq 2^{n}-t .
\end{aligned}
$$

This last inequality follows from (3.3). We obtain

$$
\begin{aligned}
|f(z)| \leq r+\sum_{j=2}^{\infty} a_{j} r^{j} & \leq r+r^{2} \sum_{j=2}^{\infty} a_{j} \\
& \leq r+r^{2}\left(\frac{2^{n}-t}{2^{n+1}-t}\right) .
\end{aligned}
$$

Similarly

$$
\begin{aligned}
|f(z)| \geq r-\sum_{j=2}^{\infty} a_{j} r^{j} & \geq r-r^{2} \sum_{j=2}^{\infty} a_{j} \\
& \geq r-r^{2}\left(\frac{2^{n}-t}{2^{n+1}-t}\right) .
\end{aligned}
$$

So,

$$
\mathrm{r}-\left(\frac{2^{\mathrm{n}}-\mathrm{t}}{2^{\mathrm{n}+1}-\mathrm{t}}\right) \mathrm{r}^{2} \leq|\mathrm{f}(\mathrm{z})| \leq \mathrm{r}+\left(\frac{2^{\mathrm{n}}-\mathrm{t}}{2^{\mathrm{n}+1}-\mathrm{t}}\right) \mathrm{r}^{2}
$$

That is 


$$
\begin{aligned}
& \mathrm{r}-\frac{2 \beta \xi(1-\alpha)}{\left(2^{\mathrm{n}+1}-2^{\mathrm{n}}\right)-\beta\left(2 \alpha \xi 2^{\mathrm{n}}-2 \xi 2^{\mathrm{n}+1}+\gamma 2^{\mathrm{n}+1}-\gamma 2^{\mathrm{n}}\right)} \mathrm{r}^{2} \leq|\mathrm{f}(\mathrm{z})| \\
& \leq \mathrm{r}+\frac{2 \beta \xi(1-\alpha)}{\left(2^{\mathrm{n}+1}-2^{\mathrm{n}}\right)-\beta\left(2 \alpha \xi 2^{\mathrm{n}}-2 \xi 2^{\mathrm{n}+1}+\gamma 2^{\mathrm{n}+1}-\gamma 2^{\mathrm{n}}\right)} \mathrm{r}^{2} .
\end{aligned}
$$

Hence the theorem.

Corollary 3.1. When $\mathrm{n}=0$, we obtain the results of Khainar and Meena More [3]

$$
\begin{array}{rl}
\mathrm{r}-\frac{2 \beta \xi(1-\alpha)}{(1+4 \beta \xi)-} & \beta(\gamma+2 \alpha \xi) \\
\mathrm{r} & \mathrm{r}-|\mathrm{f}(\mathrm{z})| \\
& \mathrm{r}+\frac{2 \beta \xi(1-\alpha)}{(1+4 \beta \xi)-\beta(\gamma+2 \alpha \xi)} \mathrm{r}^{2}
\end{array}
$$

Corollary 3.2. If $\mathrm{f}(\mathrm{z}) \in \mathrm{TS}_{0}(\alpha, \beta, \xi, 1)$, then

$$
\begin{array}{rl}
\mathrm{r}-\frac{2 \beta \xi(1-\alpha)}{(1+4 \beta \xi)-} & \beta(1+2 \alpha \xi) \\
\mathrm{r} & \mathrm{r}-|\mathrm{f}(\mathrm{z})| \\
& \mathrm{r}+\frac{2 \beta \xi(1-\alpha)}{(1+4 \beta \xi)-\beta(1+2 \alpha \xi)} \mathrm{r}^{2}
\end{array}
$$

with equality for

$$
f(z)=z-\frac{2 \beta \xi(1-\alpha)}{(1+4 \beta \xi)-\beta(1+2 \alpha \xi)} z^{2} \quad \text { at } \quad z= \pm r
$$

Corollary 3.3. If $\mathrm{f}(\mathrm{z}) \in \mathrm{TS}_{0}(\alpha, \beta, 1,1)$, then

$$
\mathrm{r}-\frac{2 \beta(1-\alpha)}{1+3 \beta-2 \alpha} \mathrm{r}^{2} \leq|\mathrm{f}(\mathrm{z})| \leq \mathrm{r}+\frac{2 \beta(1-\alpha)}{1+3 \beta-2 \alpha} \mathrm{r}^{2}
$$

and equality holds for

$$
f(z)=z-\frac{2 \beta(1-\alpha)}{1+3 \beta-2 \alpha} z^{2} \text { at } z= \pm r
$$

Theorem 3.2. If $\mathrm{f}(\mathrm{z}) \in \mathrm{TK}_{\mathrm{n}}(\alpha, \beta, \xi, \gamma)$, then

$$
\begin{aligned}
& \mathrm{r}-\frac{2 \beta \xi(1-\alpha)}{2^{\mathrm{n}+1}\left[\left(2^{\mathrm{n}+1}-2^{\mathrm{n}}\right)-\beta\left(2 \alpha \xi 2^{\mathrm{n}}-2 \xi 2^{\mathrm{n}+1}+\gamma 2^{\mathrm{n}+1}-\gamma 2^{\mathrm{n}}\right)\right]} \mathrm{r}^{2} \leq|\mathrm{f}(\mathrm{z})| \\
& \leq \mathrm{r}+\frac{2 \beta \xi(1-\alpha)}{2^{\mathrm{n}+1}\left[\left(2^{\mathrm{n}+1}-2^{\mathrm{n}}\right)-\beta\left(2 \alpha \xi 2^{\mathrm{n}}-2 \xi 2^{\mathrm{n}+1}+\gamma 2^{\mathrm{n}+1}-\gamma 2^{\mathrm{n}}\right)\right]} \mathrm{r}^{2} .
\end{aligned}
$$

Proof. The proof of this theorem is analogous to that of Theorem 3.1 because a function $\mathrm{f}(\mathrm{z}) \in \mathrm{TK}_{\mathrm{n}}(\alpha, \beta, \xi, \gamma)$ if and only if $\mathrm{zf}^{\prime}(\mathrm{z}) \in \mathrm{TS}_{\mathrm{n}}(\alpha, \beta, \xi, \gamma)$. So it is enough to replace $\mathrm{a}_{\mathrm{j}}$ by $\mathrm{j}^{\mathrm{n}+1} \mathrm{a}_{\mathrm{j}}$ in Theorem 3.1 .

Corollary 3.4. When $\mathrm{n}=0$, we obtain the results of Khainar and Meena More [3]

$$
\mathrm{r}-\frac{\beta \xi(1-\alpha)}{(1+4 \beta \xi)-\beta(\gamma+2 \alpha \xi)} \mathrm{r}^{2} \leq|\mathrm{f}(\mathrm{z})| \leq \mathrm{r}+\frac{\beta \xi(1-\alpha)}{(1+4 \beta \xi)-\beta(\gamma+2 \alpha \xi)} \mathrm{r}^{2}
$$

Corollary 3.5. If $\mathrm{f}(\mathrm{z}) \in \mathrm{TK}_{0}(\alpha, \beta, \xi, 1)$, then

$$
\mathrm{r}-\frac{\beta \xi(1-\alpha)}{(1+4 \beta \xi)-\beta(1+2 \alpha \xi)} \mathrm{r}^{2} \leq|\mathrm{f}(\mathrm{z})| \leq \mathrm{r}+\frac{\beta \xi(1-\alpha)}{(1+4 \beta \xi)-\beta(1+2 \alpha \xi)} \mathrm{r}^{2}
$$

with equality holds for

$$
\mathrm{f}(\mathrm{z})=\mathrm{z}-\frac{\beta \xi(1-\alpha)}{(1+4 \beta \xi)-\beta(1+2 \alpha \xi)} \mathrm{z}^{2} \text { at } \mathrm{z}= \pm \mathrm{r} .
$$

This result is due to [1] and [4].

Corollary 3.6. If $\mathrm{f}(\mathrm{z}) \in \mathrm{TK}_{0}(\alpha, \beta, 1,1)$, then

$$
r-\frac{\beta(1-\alpha)}{1+3 \beta-2 \alpha} r^{2} \leq|f(z)| \leq r+\frac{\beta(1-\alpha)}{1+3 \beta-2 \alpha} r^{2}
$$

with equality holds for

$$
f(z)=z-\frac{\beta(1-\alpha)}{1+3 \beta-2 \alpha} z^{2} \text { at } z= \pm r .
$$

This result is due to [5].

Theorem 3.3. If $\mathrm{f}(\mathrm{z}) \in \mathrm{TS}_{\mathrm{n}}(\alpha, \beta, \xi, \gamma)$, then

$$
\begin{aligned}
& 1-\frac{4 \beta \xi(1-\alpha)}{\left(2^{\mathrm{n}+1}-2^{\mathrm{n}}\right)-\beta\left(2 \alpha \xi 2^{\mathrm{n}}-2 \xi 2^{\mathrm{n}+1}+\gamma 2^{\mathrm{n}+1}-\gamma 2^{\mathrm{n}}\right)} \mathrm{r} \leq\left|\mathrm{f}^{\prime}(\mathrm{z})\right| \\
& \leq 1+\frac{4 \beta \xi(1-\alpha)}{\left(2^{\mathrm{n}+1}-2^{\mathrm{n}}\right)-\beta\left(2 \alpha \xi 2^{\mathrm{n}}-2 \xi 2^{\mathrm{n}+1}+\gamma 2^{\mathrm{n}+1}-\gamma 2^{\mathrm{n}}\right)} \mathrm{r}
\end{aligned}
$$

Proof. Since $\mathrm{f}(\mathrm{z}) \in \mathrm{TS}_{\mathrm{n}}(\alpha, \beta, \xi, \gamma)$, we have

$$
\sum_{j=2}^{\infty}\left(j^{n+1}-t\right) a_{j} \leq j^{n}-t
$$

Where $\mathrm{t}=\mathrm{j}^{\mathrm{n}}-\frac{2 \beta \xi(1-\alpha) \mathrm{j}^{\mathrm{n}}}{1+2 \beta \xi-\gamma \beta}$.

In view of Theorem 3.1, we have

$$
\begin{aligned}
\sum_{j=2}^{\infty} j^{n+1} a_{j} & =\sum_{j=2}^{\infty}\left(j^{n+1}-t\right) a_{j}+t \sum_{j=2}^{\infty} a_{j} \\
& \leq\left(j^{n}-t\right)+t\left(\frac{j^{n}-t}{j^{n+1}-t}\right)
\end{aligned}
$$

When $\mathrm{j}=2$,

$$
\sum_{j=2}^{\infty} j^{n+1} a_{j} \leq\left(2^{n}-t\right)+t\left(\frac{2^{n}-t}{2^{n+1}-t}\right)
$$

Therefore

$$
\begin{aligned}
\left|f^{\prime}(z)\right| & \leq 1+\sum_{j=2}^{\infty} j a_{j}|z|^{j-1} \\
& \leq 1+r\left[\left(2^{n}-t\right)+t\left(\frac{2^{n}-t}{2^{n+1}-t}\right)\right]
\end{aligned}
$$

Similarly

$$
\left|f^{\prime}(z)\right| \geq 1-r\left[\left(2^{n}-t\right)+t\left(\frac{2^{n}-t}{2^{n+1}-t}\right)\right]
$$

So

$$
1-r\left[\left(2^{n}-t\right)+t\left(\frac{2^{n}-t}{2^{n+1}-t}\right)\right] \leq\left|f^{\prime}(z)\right| \leq 1+r\left[\left(2^{n}-t\right)+t\left(\frac{2^{n}-t}{2^{n+1}-t}\right)\right]
$$

Substituting $\mathrm{t}$ we have 


$$
\begin{aligned}
& 1-\frac{4 \beta \xi(1-\alpha)}{\left(2^{\mathrm{n}+1}-2^{\mathrm{n}}\right)-\beta\left(2 \alpha \xi 2^{\mathrm{n}}-2 \xi 2^{\mathrm{n}+1}+\gamma 2^{\mathrm{n}+1}-\gamma 2^{\mathrm{n}}\right)} \mathrm{r} \leq\left|\mathrm{f}^{\prime}(\mathrm{z})\right| \\
& \leq 1+\frac{4 \beta \xi(1-\alpha)}{\left(2^{\mathrm{n}+1}-2^{\mathrm{n}}\right)-\beta\left(2 \alpha \xi 2^{\mathrm{n}}-2 \xi 2^{\mathrm{n}+1}+\gamma 2^{\mathrm{n}+1}-\gamma 2^{\mathrm{n}}\right)} \mathrm{r}
\end{aligned}
$$

Corollary 3.7. When $\mathrm{n}=0$, we obtain the results of Khainar and Meena More [3]

$$
1-r\left(\frac{2(1-t)}{2-t}\right) \leq\left|f^{\prime}(z)\right| \leq 1+r\left(\frac{2(1-t)}{2-t}\right)
$$

Substituting t, from (3.5) in (3.6), we obtain

$$
1-\frac{4 \beta \xi(1-\alpha)}{(1-\gamma \beta)+2 \beta \xi(2-\alpha)} \mathrm{r} \leq\left|\mathrm{f}^{\prime}(\mathrm{z})\right| \leq 1+\frac{4 \beta \xi(1-\alpha)}{(1-\gamma \beta)+2 \beta \xi(2-\alpha)} \mathrm{r}
$$

Corollary 3.8. If $\mathrm{f}(\mathrm{z}) \in \mathrm{TS}_{0}(\alpha, \beta, \xi, 1)$, then

$$
1-\frac{4 \beta \xi(1-\alpha)}{(1-\beta)+2 \beta \xi(2-\alpha)} \mathrm{r} \leq\left|\mathrm{f}^{\prime}(\mathrm{z})\right| \leq 1+\frac{4 \beta \xi(1-\alpha)}{(1-\beta)+2 \beta \xi(2-\alpha)} \mathrm{r}
$$

Corollary 3.9. If $\mathrm{f}(\mathrm{z}) \in \mathrm{TS}_{0}(\alpha, \beta, 1,1)$, then

$$
1-\frac{4 \beta(1-\alpha)}{1+3 \beta-2 \alpha} \mathrm{r} \leq\left|\mathrm{f}^{\prime}(\mathrm{z})\right| \leq 1+\frac{4 \beta(1-\alpha)}{1+3 \beta-2 \alpha} \mathrm{r}
$$

Theorem 3.4. If $\mathrm{f}(\mathrm{z}) \in \mathrm{TK}_{\mathrm{n}}(\alpha, \beta, \xi, \gamma)$, then

$$
\begin{aligned}
& 1-\frac{4 \beta \xi(1-\alpha)}{2^{\mathrm{n}+1}\left[\left(2^{\mathrm{n}+1}-2^{\mathrm{n}}\right)-\beta\left(2 \alpha \xi 2^{\mathrm{n}}-2 \xi 2^{\mathrm{n}+1}+\gamma 2^{\mathrm{n}+1}-\gamma 2^{\mathrm{n}}\right)\right]} \mathrm{r} \leq\left|\mathrm{f}^{\prime}(\mathrm{z})\right| \\
& \leq 1+\frac{4 \beta \xi(1-\alpha)}{2^{\mathrm{n}+1}\left[\left(2^{\mathrm{n}+1}-2^{\mathrm{n}}\right)-\beta\left(2 \alpha \xi 2^{\mathrm{n}}-2 \xi 2^{\mathrm{n}+1}+\gamma 2^{\mathrm{n}+1}-\gamma 2^{\mathrm{n}}\right)\right]} \mathrm{r}
\end{aligned}
$$

Proof. The proof of this theorem is similar to that of Theorem 3.3, because a function $\mathrm{f}(\mathrm{z}) \in \mathrm{TK}_{\mathrm{n}}(\alpha, \beta, \xi, \gamma)$ if and only if $\mathrm{zf}^{\prime}(\mathrm{z}) \in \operatorname{TS}_{\mathrm{n}}(\alpha, \beta, \xi, \gamma)$. i.e., replacing $\mathrm{a}_{\mathrm{j}}$ by $\mathrm{j}^{\mathrm{n}+1} \mathrm{a}_{\mathrm{j}}$ in Theorem 3.3.

Corollary 3.10. When $\mathrm{n}=0$, we obtain the results of Khainar and Meena More [3]

$$
1-\frac{2 \beta \xi(1-\alpha)}{(1-\gamma \beta)+2 \beta \xi(2-\alpha)} \mathrm{r} \leq\left|\mathrm{f}^{\prime}(\mathrm{z})\right| \leq 1+\frac{2 \beta \xi(1-\alpha)}{(1-\gamma \beta)+2 \beta \xi(2-\alpha)} \mathrm{r}
$$

Corollary 3.11. If $\mathrm{f}(\mathrm{z}) \in \mathrm{TK}_{0}(\alpha, \beta, \xi, 1)$, then

$$
1-\frac{2 \beta \xi(1-\alpha)}{(1-\beta)+2 \beta \xi(2-\alpha)} \mathrm{r} \leq\left|\mathrm{f}^{\prime}(\mathrm{z})\right| \leq 1+\frac{2 \beta \xi(1-\alpha)}{(1-\beta)+2 \beta \xi(2-\alpha)} \mathrm{r}
$$

Corollary 3.12. If $\mathrm{f}(\mathrm{z}) \in \mathrm{TK}_{0}(\alpha, \beta, 1,1)$, then

$$
1-\frac{2 \beta(1-\alpha)}{1+3 \beta-2 \alpha} \mathrm{r} \leq\left|\mathrm{f}^{\prime}(\mathrm{z})\right| \leq 1+\frac{2 \beta(1-\alpha)}{1+3 \beta-2 \alpha} \mathrm{r}
$$

IV. EXTREME POINTS FOR TSN $(\alpha, \beta, \xi, \gamma)$

The determination of the extreme points of a family of univalent functions enables us to solve many extremal problems.
Theorem 4.1. Let $\mathrm{f}_{1}(\mathrm{z})=\mathrm{z}$ and

$$
\begin{aligned}
& \mathrm{f}_{\mathrm{j}}(\mathrm{z})=\frac{2 \beta \xi(1-\alpha)}{\left(\mathrm{j}^{\mathrm{n}+1}-\mathrm{j}^{\mathrm{n}}\right)-\beta\left(2 \alpha \xi \mathrm{j}^{\mathrm{n}}-2 \xi \mathrm{j}^{\mathrm{n}+1}+\gamma \mathrm{j}^{\mathrm{n}+1}-\gamma \mathrm{j}^{\mathrm{n}}\right)} \mathrm{z}^{\mathrm{j}} \\
& \quad \text { for } \mathrm{j}=2,3,4, \ldots
\end{aligned}
$$

Then $\mathrm{f}(\mathrm{z}) \in \mathrm{TK}_{\mathrm{n}}(\alpha, \beta, \xi, \gamma)$ if and only if $\mathrm{f}(\mathrm{z})$ can be expressed in the forms

$$
f(z)=\sum_{j=1}^{\infty} \lambda_{j} f_{j}(z)
$$

Where $\lambda_{\mathrm{j}} \geq 0$ and $\sum_{\mathrm{j}=1}^{\infty} \lambda_{\mathrm{j}}=1$

Proof. Let $\mathrm{f}(\mathrm{z})=\sum_{\mathrm{j}=1}^{\infty} \lambda_{\mathrm{j}} \mathrm{f}_{\mathrm{j}}(\mathrm{z}), \quad \lambda_{\mathrm{j}} \geq 0, \mathrm{j}=1,2, \ldots$ with $\sum_{\mathrm{j}=1}^{\infty} \lambda_{\mathrm{j}}=1$

$$
\begin{aligned}
& f(z)=\sum_{j=1}^{\infty} \lambda_{j} f_{j}(z) \\
& =\lambda_{1} f_{1}(z)+\sum_{j=2}^{\infty} \lambda_{j} f_{j}(z) \\
& =\lambda_{1}(z)+\sum_{j=2}^{\infty} \lambda_{j}\left[z-\frac{2 \beta \xi(1-\alpha)}{\left(j^{n+1}-j^{n}\right)-\beta\left(2 \alpha \xi j^{n}-2 \xi j^{n+1}+\gamma j^{n+1}-\gamma j^{n}\right)} z^{j}\right]
\end{aligned}
$$

that is,

$f(z)=z-\sum_{j=2}^{\infty} \lambda_{j} \frac{2 \beta \xi(1-\alpha)}{\left(j^{n+1}-j^{n}\right)-\beta\left(2 \alpha \xi j^{n}-2 \xi j^{n+1}+\gamma j^{n+1}-\gamma j^{n}\right)} z^{j}$

Then

$$
\begin{aligned}
\sum_{j=2}^{\infty} \lambda_{j} & \frac{2 \beta \xi(1-\alpha)}{\left[\left(j^{n+1}-j^{n}\right)-\beta\left(2 \alpha \xi j^{n}-2 \xi j^{n+1}+\gamma j^{n+1}-\gamma j^{n}\right)\right]} \\
& \times \frac{\left(j^{n+1}-j^{n}\right)-\beta\left(2 \alpha \xi j^{n}-2 \xi j^{n+1}+\gamma j^{n+1}-\gamma j^{n}\right)}{2 \beta \xi(1-\alpha)} \\
\sum_{j=2}^{\infty} \lambda_{j} & =1-\lambda_{1} \leq 1 .
\end{aligned}
$$

$\therefore \mathrm{f}(\mathrm{z}) \in \mathrm{TK}_{\mathrm{n}}(\alpha, \beta, \xi, \gamma)$.

Conversely, suppose $\mathrm{f}(\mathrm{z}) \in \mathrm{TK}_{\mathrm{n}}(\alpha, \beta, \xi, \gamma)$, then remark of Theorem 2.1 gives us

$$
a_{j} \leq \frac{2 \beta \xi(1-\alpha)}{\left(j^{n+1}-j^{n}\right)-\beta\left(2 \alpha \xi j^{n}-2 \xi j^{n+1}+\gamma j^{n+1}-\gamma j^{n}\right)}
$$

we take

$$
\lambda_{j}=\frac{\left(j^{n+1}-j^{n}\right)-\beta\left(2 \alpha \xi j^{n}-2 \xi j^{n+1}+\gamma j^{n+1}-\gamma j^{n}\right)}{2 \beta \xi(1-\alpha)} a_{j}, j=2,3,4, \ldots
$$

and $\lambda_{1}=1-\sum_{j=2}^{\infty} \lambda_{j}$. 


$$
\text { Then } f(z)=\sum_{j=1}^{\infty} \lambda_{j} f_{j}(z) \text {. }
$$

Corollary 4.1. When $\mathrm{n}=0$, we obtain the result of Khairnar and Meena More [3]. That is, $\mathrm{f}_{1}(\mathrm{z})=\mathrm{z}$,

$$
f_{j}(z)=\frac{2 \beta \xi(1-\alpha)}{(j-1)-\beta(2 \alpha \xi-2 \xi j+\gamma j-\gamma)}, \text { for } j=2,3, \ldots
$$

Corollary 4.2. If $\mathrm{f}_{1}(\mathrm{z})=\mathrm{z}$ and

$$
f_{j}(z)=z-\frac{2 \beta \xi(1-\alpha)}{(j-1)-\beta(2 \alpha \xi-2 \xi j+j-1)} z^{j} \text { for } j=2,3, \ldots
$$

Then $\mathrm{f}(\mathrm{z}) \in \mathrm{TK}_{\mathrm{n}}(\alpha, \beta, \xi, 1)$ if and only if, $\mathrm{f}(\mathrm{z})$ can be expressed in the form,

$$
f(z)=\sum_{j=1}^{\infty} \lambda_{j} f_{j}(z), \text { where } \lambda_{j} \geq 0, j=1,2, \ldots, \sum_{j=1}^{\infty} \lambda_{j}=1 .
$$

This result is due to [1] and [4].

Corollary 4.3. If $\mathrm{f}_{1}(\mathrm{z})=\mathrm{z}$ and

$$
f_{j}(z)=z-\frac{2 \beta(1-\alpha)}{(j-1)-\beta(2 \alpha-j-1)} z^{j} \text { for } j=2,3, \ldots
$$

Then $\mathrm{f}(\mathrm{z}) \in \mathrm{TK}_{\mathrm{n}}(\alpha, \beta, 1,1)$ if and only if, $\mathrm{f}(\mathrm{z})$ can be expressed in the form,

$$
f(z)=\sum_{j=1}^{\infty} \lambda_{j} f_{j}(z), \text { where } \lambda_{j} \geq 0, j=1,2, \ldots, \sum_{j=1}^{\infty} \lambda_{j}=1 .
$$

This result is due to Kulkarni [4].

Corollary 4.4. If $\mathrm{f}_{1}(\mathrm{z})=\mathrm{z}$ and

$$
f_{j}(z)=z-\frac{z^{j}}{j} .
$$

Then $\mathrm{f}(\mathrm{z}) \in \mathrm{TK}_{\mathrm{n}}(0,1,1,1)$ if and only if, $\mathrm{f}(\mathrm{z})$ can be expressed in the form,

$$
f(z)=\sum_{j=1}^{\infty} \lambda_{j} f_{j}(z), \text { where } \lambda_{j} \geq 0, j=1,2, \ldots, \sum_{j=1}^{\infty} \lambda_{j}=1
$$

\section{CONCLUSION}

In this paper making use of the Salagean differential operator, new subclasses of analytic and univalent functions are introduced for the functions with negative coefficients. Many subclasses which are already studied by various researchers are obtained as special cases of our two new subclasses. We have obtained various properties such as coefficient estimates, growth and distortion theorem for the class of functions. Further new subclasses may be possible from the two classes introduced in this paper.

\section{REFERENCES}

[1] R. Aghalary and S. Kulkarni, "Some theorems on univalent functions", J. Indian Acad. Math., Vol. 24, No. 1, Pp. 81-93, 2002.

[2] L. de Branges, A proof of Bieberbach conjecture, Acta Math., Vol. 154, No. 1-2, Pp. 137-152, 1985.

[3] S.M. Khairnar and Meena More, "Certain family of analytic and univalent functions with normalized conditions", Acta Mathematica Academiae Paedagogicae, Vol. 24, Pp. 333-344, 2008.
[4] S.R. Kulkarni, "Some problems connected with univalent functions", $\mathrm{Ph} . \mathrm{D}$. Thesis, Shivaji University, Kolhapur, 1981.

[5] S. Owa and J. Nishiwaki, "Coefficient Estimates for certain classes of analytic functions", J. Inequal. Pure Appl. Math., Vol. 3, No. 5, Article 72, 5pp (electonic), 2002.

[6] G.S. Salagean, "Subclasses of univalent functions", Lecture Notes in Mathematics, Volume 1013, Springer-Verlag, Berlin, Pp. 362-372, 1983.

[7] H. Silverman, "Univalent functions with negative coefficients", Proc. Amer. Math. Soc., Vol. 51, Pp. 109-116, 1975.

[8] H. Silverman and E. Silvia, "Subclasses of prestarlike functions", Math. Japon, Vol. 29, No. 6, Pp. 929-935, 1984. 